DOE/ER/45267--6

DE92 016313

\title{
SURFACES AND THIN FILMS STUDIED
}

BY PICOSECOND ULTRASONICS

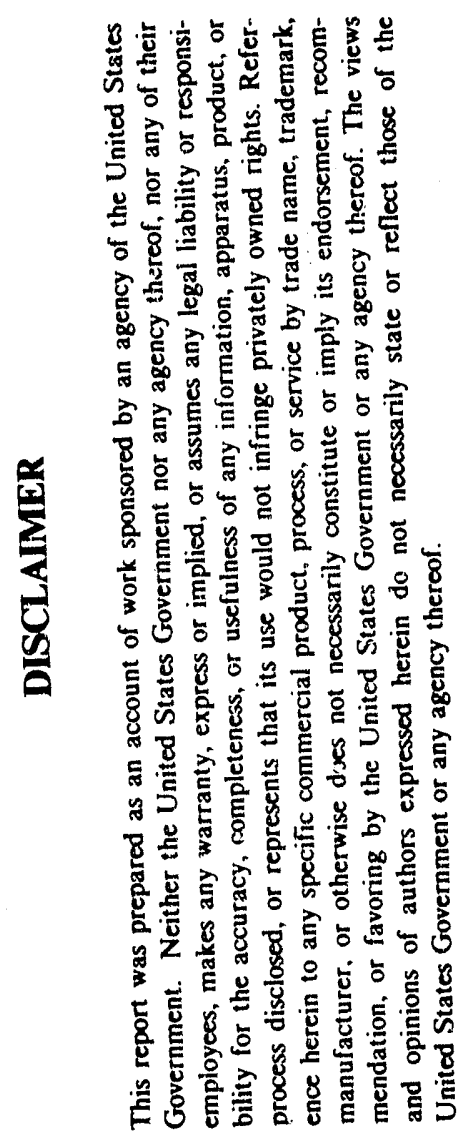

\author{
Progress Report
}

for Period December 1, 1989 - November 30, 1992

Humphrey J. Maris and Jan Tauc

Brown University

Providence, Rhode Island 02912

May 1992

Prepared for

THE U.S. DEPARTMENT OF ENERGY

AGREEMENT NO. DE--FG02-ER45267 


\section{SUMMARY OF RESEARCH IN GRANT PERIOD BEGINNING 12/1/89}

The research that is supported by DOE grant DE-FG-02-86ER45267 is the study of thin films and interfaces via the use of the picosecond ultrasonic technique. In these experiments ultrasonic waves are excited in a structure by means of a picosecond light pulse ("pump pulse"). The propagation of these waves is detected through the use of a probe light pulse that is tim?-delayed relative to the pump. This probe pulse measures the change $\Delta R(t)$ in the optical reflectivity of the structure that occurs because the ultrasonic wave changes the optical properties of the structure. This technique make possible the study of the attenuation and velocity of ultrasonic waves up to much higher frequencies than was previously possible (up to at least $500 \mathrm{GHz}$ ). In addition, the excellent time-resolution of the method makes it possible to study nanostructures of linear dimensions down to $100 \AA$ or less by ultrasonic pulse-echo techniques.

\section{a) PICOSECOND ULTRASONICS FACILITY}

During the present grant period we were fortunate to receive from the Department of Energy a University Research Instrumentation grant for the establishment of a state-ofthe-art picosecond ultrasonics facility. This grant was awarded in September 1990 and in the time since then a substantial part of the effort of our group has been directed toward the establishment of this facility. For the past six months the facility has been essentially complete and we have been able to mike use of the facility to study a range of problems of interest.

To investigate physical properties bv the picosecond ultrasonic trchnique it is essential to be able to measure accurately the very small photo-induced changes iri optical reflectivity associated with the ultrasonic echoes. The development of an overall system to achieve this was the primary goal governing the design of the facility. The main elements of the facility are the following:

Laser System: The light source for the experiments is an Antares YAG laser that pumps a Satori dye iaser (both lasers purchased from Coherent Inc). The YAG produces $100 \mathrm{psec}$ pulses of wavelength $1.06 \mu$ and average power $22 \mathrm{~W}$. These pulses are monitored on a Tektronix sampling scope using an ultrafast photodiode. The YAG output is frequencydoubled to produce $75 \mathrm{psec}, 532 \mathrm{~nm}$, pulses of mean power $2 \mathrm{~W}$, which pump the Satori. The output of the Satori is tunable between 620 and $660 \mathrm{~nm}$ using Kyton red dye. The pulse width is typically 175 fsec with a repetition rate of $76 \mathrm{MHz}$ and the mean power is $300 \mathrm{~mW}$. The pulse width is monitored by means of an Irrad autocorrelator.

Modulation and Measurement Technique: The laser output is split into pump and probe beams with an intensity ratio of about 10 to 1 . The pump beam can be modulated at up to $20 \mathrm{MHz}$ by means of an acousto-optic modulator, but the modulation frequency 
is usually chosen to be $1 \mathrm{MHz}$ because of the favorable characteristics of the detector electronics in the range of this frequency. The probe beam is time-delayed by a computercontrolled delay stage driven by a de servo motor. This stage provides a delay of up to 3 nsecs which is controllable in steps of 7.5 fsecs. Because the main source of noise in the measurement comes from the random variations in intensity of the probe beam, we have developed a technique that substantially reduces the effect of these fluctuations. Part of the probe beam is directed onto the sample and after reflection this is detected by a photodiode "A". The remaining part of the beam goes directly to another photodiode "B". The outputs of "A" and "B" are amplified so as to make their low frequency components equal, and then "B" is subtracted from " $\mathrm{A}$ " to leave primarily the $1 \mathrm{MHz}$ signal. In favorable cases this arrangement has led to a $50 \mathrm{~dB}$ reduction in noise. To demodulate the $1 \mathrm{MHz}$ signal we first mix it with a stable frequency source in an ultra-low-noise mixer to lower the frequency to about $20 \mathrm{kHz}$. Final demodulation is achieved using a Stanford Inc lock-in amplifier. A PC clone together with LABWINDOWS software is used for control of the system and for storing and manipulating the averaged signals.

Cryostats: To make routine measurements at temperatures below room temperature we purchased a Janis Supertran continuous flow cryostat together with a Lake Shore temperature controller. As discussed in the section on proposed work, we also have a need for a more sophisticated cryostat that will enable a wider variety of experiments to be performed and which will reach lower temperatures. We have begun construction of a cryostat that will reach a temperature of $1.5 \mathrm{~K}$, and this cryostat will also enable measurements to be made on samples prepared in situ in the cryostat.

We have installed this apparatus in a separate new laboratory prepared using university funds. The laboratory is provided with an air cleaning system to maintain the operation of the optical system. The overall sensitivity of this system is approximately 10 times superior to our old system and the system has proven substantially more reliable. This improvement in performance makes a vory substantial difference to our experimental program. It enables us to study samples in which the changes in reflectivity were previously ton small to be measured, and makes possible the resolution of small but important features in the response. In addition, for any given sample a comparable signal-to-noise ratio can be achieved with the new system in roughly one hundredth of the time that was required with the old system. Thus the new system has substantially increased the productivity of our group and the range of problems that can be investigated.

\section{b) NONDESTRUCTIVE EVALUATION OF NANOSTRUCTURES}

As mentioned above the picosecond ultrasonics technique has opened up exciting possibilities for the study of the mechanical properties of very small structures. At the time of our previous proposal we described a series of experiments that we planned to perform in collaboration with three groups at IBM, Yorktown Heights. The goal was to study systems that were well-characterized by other means so that our results could be correlated with other measurements. In addition, we wanted to attempt some projects 
that were not just demonstrations of the method, but which addressed practical problems of current interest that could not readily be solved by other techniques.

These projects have worked out very well. The first set of experiments were studies of titanium silicide formation and were performed in collaboration with the group of Dr. Jim Harper. The C54 phase of $\mathrm{TiSi}_{2}$ is the equilibrium phase and because of its low resistivity is well-suited for device contact metallization in VLSI technology. The transformation from the high resistivity metastable $\mathrm{C} 49$ phase normally occurs at an annealing temperature of about $700^{\circ} \mathrm{C}$, but is influenced by impurities. Thus, a non-destructive probe of the transformation is desirable. We made ultrasonic measurements on a series of samples consisting of a $360 \AA \mathrm{Ti}$ film deposited onto a $\mathrm{Si}$ substrate and annealed at a sequence of different temperatures. The measured $\Delta R(t)$ had a qualitatively different form for the unreacted $\mathrm{Ti}$ and for the different silicide phases, and thus the method is capable of monitoring the phase transitions $[14,16]$.

The goal of the second project has been the use of picosecond ultrasonics to detect thin layers of contaminants at buried interfaces. Such contaminants weaken the bonding at an interface and can, under the influence of thermal and mechanical stress, lead to delamination. Buried interfaces can be studied by various techniques including crosssectional SEM and TEM, but of course these are destructive techniques. One way in which interfacial contamination can occur in real VLSI systems is through the formation of polymeric fluorocarbon $\left(\mathrm{CF}_{\boldsymbol{x}}\right)$ layers during reactive ion etching processes of silicon. This process has been extensively studied by the group of Drs. G.W. Rubloff and G.S. Oehrlein at IBM. If a metallic film is deposited onto silicon that has a surface layer of $\mathrm{CF}_{\boldsymbol{x}}$ contamilant, the adhesion of the metal film will be poor. We have used the picosecond optics technique to study a series of samples prepared for us by the IBM group. The experiment is described in one of the reprints [15] attached at the back of this report. The metal film is aluminium of thickness $\sim 150 \AA$. For very thin films such as this the effect of the pump light pulse is to set the film into vibration in its fundamental thickness vibration. The vibration is damped by the transmission of energy into the substrate. Thus the presence of the thin contaminant layer reduces the damping rate of the $\mathrm{Al}$ film. We measured this damping rate for $\mathrm{Al}$ films on a bare substrate and with intervening layers of $\mathrm{CF}_{x}$ of thickness 5 and $20 \AA$.

These data showed that a contaminant layer as thin as $5 \AA$ can readily be detected in this way. However, a detailed analysis revealed surprising results $[15,17]$. It is straightforward to use elasticity theory together with the literature values for the elastic properties of $\mathrm{CF}_{\boldsymbol{x}}$ to calculate what the damping rate of the aluminium vibration should be. We found that the measured damping for the $5 \AA$ sample is, in fact, considerably larger than expected theoretically, i.e. the $\mathrm{CF}_{\boldsymbol{x}}$ layer is much stiffer than expected. We believe that this discrepancy comes about because the mechanics of a $5 \AA$ layer are not adequately described by standard continuum elasticity theory. For example, the "softness" of a macroscopic polymer comes about because of the weakness of the van der Waal's forces between the polymer units and the limited amount of cross-linking. For a $5 \AA$ layer there will be molecular units that run directly from the $\mathrm{Si}$ surface to the $\mathrm{Al}$ film and these will give the 
$\mathrm{CF}_{x}$ layer a greater stiffness than would be expected based on macroscopic elasticity. This work will be continued to study in more detail the effect of varying the $\mathrm{CF}_{\boldsymbol{x}}$ thickness.

The structures discussed so far have been planar. In a third project we have studied the much more complicated problem of the vibrations of laterally-patterned nanostructures. In this project the goal has been to see if we can use the picosecond ultrasonic technique to measure the frequencies of the principal normal morles of vibratic $n$ of such structures, and then to compare the results with frequencies calculated based on what is known about the geometry of the structure. The samples consisted of gold stripes or dots deposited onto fused quartz substrates, and were prepared for us by the group of Dr. Trey Smith at IBM. The experiment and the results are described in detail in the attached preprint [18]. We have performed a detailed analysis of these results [18] and similar data on other stripe and dot samples, and have been able to achieve a complete understanding of the origin of the rather complex features that are seen. In this experiment the pump light pulse is absorbed in the gold bars and sets them into vibration. The oscillating elastic strain in the gold causes a modulation of the optical reflectivity at the normal mode frequencies of the gold bars. Thus, by identifying these frequencies as having this origin we have been able to measure some of these normal mode frequencies. We have compared the results with frequencies calculated by a finite-element normal- mode analysis of the nanostructure[18] and have obtained excellent agreement. The finite-element analysis also gives the displacement pattern associated with each mode (see preprint). For the particular samples that we have studied there is only a small coupling between the vibrations on different bars, and so to a good approximation one can consider that what we are observing is the vibration of individual bars (as in a xylophone!). The main way in which these vibrations lose energy is via acoustic radiation into the substrate. By an analysis of the measured $\Delta R(t)$ we can determine the damping rate, and we find that this is in excellent agreement with the theoretical results.

In addition to this signal that comes from the vibrations of the gold bars, there are other types of oscillation cletected in $\Delta R(t)$ that originate from the scattering of the probe light by the elastic waves that are radiated into the substrate. To work out a satisfactory theory it is necessary to allow for the fact that each gold bar launches a cylindrical elastic wave into the substrate, and to allow for the wide variety of paths that the probe light can take before and after being scattered at the elastic wave. The results of this calculation are in excellent agreement with the experimental results.

We have recently started a project to measure the sound velocity and attenuation in $\mathrm{C}_{60}$ ilms prepared for us by Dr. Art Hebard at ATT. We plan to compare the results of these measurements, which will be at an ultrasonic frequency of 10 to $100 \mathrm{GHz}$, with recent data obtained by the use of the vibrating-reed technique at frequencies of 10 to $20 \mathrm{kHz}$, in order to better understand structural relaxation in this material. The low frequency data reveal a peak in the sound attenuation in the region around $160 \mathrm{I}$ together with a change in sound velocity. These features have been attributed to structural relaxation, and by making a measurements at much higher frequencies we will be able to learn more about this relaxation process. 


\section{c) STUDIES OF AMORPHOUS MATERIALS}

The research that we have performed under the present DOE grant has been an investigation of the attenuation of high frequency ultrasonic waves in amorphous materials, or equivalently the study of the mean-free path of longitudinal phonons. The major goal of this work is to use these measurements, combined with existing data on thermal conductivity, to achieve a microscopic understanding of how heat flows in amorphous materials, and how universal features such as the "plateau" in the thermal conductivity arise.

During the current grant period we have completed a set of measurements of the attenuation in amorphous $\mathrm{SiO}_{2}$. We were able to cover the frequency range from 30 $\mathrm{GHz}$ to $440 \mathrm{GHz}$, and made measurements from room temperature down to $80 \mathrm{~K}$. For the measurements at $30 \mathrm{GHz}$ we used a special technique "picosecond acoustic interferometry" that we have developed $[4,8]$, that has the advantage that a bulk sample can be used, rather than a thin film. We performed a detailed analysis of this method [8] and were able to make accurate measurements of the attenuation and sound velocity (see attached reprint [4]). These results were found to be in good agreement with earlier measurements at the same frequency by Vacher et al who used the Brillouin scattering technique. To make measurements at higher frequencies we used the pulse-echo technique with thin film a- $\mathrm{SiO}_{2}$ samples of thickness down to $280 \AA$. For a substrate we used single crystal tungsten. This substrate has the great advantage that, since the acoustic impedance of $\mathrm{W}$ is so large, the loss of the sound wave due to transmission from the $\mathrm{a}^{--} \mathrm{SiO}_{2}$ into the $\mathrm{W}$ is small and easily corrected for. The experiments and the results are described in more detail in the attached eprint [9]. Between 80 and $300 \mathrm{~K}$ the attenuation is almost independent of temperature. The frequency-dependence appears to be slightly slower than quadratic up to $200 \mathrm{GHz}$, ind possibly faster than quadratic above this frequency. At the highest frequency (440 $\left.x_{\mathrm{Hz}}\right)$ the mean-free-path $\Lambda(\nu, T)$ was found to be $\sim 110 \AA$. For comparison the phonon wavelength at this same frequency is $130 \AA$. Thus the wave is very heavily damped, but not to the point that the Ioffé-Regel condition for localization $(k \Lambda \approx 1, k=$ wave vector $)$ isi close to being satisfied since $k \Lambda$ is still $\approx 6$.

Several groups (including R. Orbach and coworkers, and Graebner, Golding and Allen) 1 ave proposed that the peculiar temperature-dependence of the thermal conductivity of a. norphous materials can be understood if phonons above a certain critical frequency are ls calized. Our measurements show that if localization does occur, the localization frequency in $\mathrm{SiO}_{2}$ must be greater than our highest frequency of $440 \mathrm{GHz}$. To search for lo alization it is of interest to look at a material in which the plateau occurs at a lower te:mperature, since the same type of analysis of the thermal conductivity then leads to the resu lt that localization should occur at a lower frequency. It was for this reason that we pr posed to study the attenuation in the polymer $\mathrm{CF}_{x}$. We have received some samples from Rubloff's group at IBM. We have so far made preliminary measurements at one freque icy $(\sim 200 \mathrm{GHz})$. The results are remarkable: they indicate that the mean-free-path at th is frequency is in the range 15 to $20 \AA$. This is substantially less than the wavelength of 
$70 \AA$, but still does not strictly satisfy the Ioffé-Regel criterion for localization. We again see no significant change in attenuation down to $80 \mathrm{~K}$. We will receive a more complete set of samples from IBM shortly, and propose to make measurements to cover the range 10 to $300 \mathrm{GHz}$.

We currently have no idea as to what can cause such a strong scattering. Even allowing for the disorder intrinsic to a polymer, it is hard to understand how a wave can be attenuated in a distance that is less than one third of its wavelength.

\section{d) MEASUREMENTS OF HEAT FLOW BETWEEN FILMS AND CRYSTALS}

Kapitza was the first to show that when heat flows across the interface between two materials a temperature jump $\Delta T$ occurs at the boundary. This jump is related to the heat current $\dot{Q}$ by the relation

$$
\Delta T=\frac{\dot{Q} R_{K}}{A}
$$

where $A$ is the area of the interface and $R_{K}$ is now called the Kapitza resistance. This resistance arises because some of the thermal phonons that attempt to cross the boundary are reflected due to the different properties (e.g. atomic mass) of the two media. This resistance was originally observed by Kapitza in the course of some experiments with superfluid helium, and it is easy to observe in this system because the resistance is large. The resistance is not important when considering the flow of heat between two macroscopic solids at room temperature, because it normally appears in series with the bulk resistance of the materials. However, in very small structures such as occur in VLSI the Kapitza resistance plays an important role in determining the heat flow.

This resistance can be determined from an analysis of the background contribution to the measured change $\Delta R(t)$ in the optical reflectivity. $\Delta R(t)$ consists of sharp features originating from the acoustic echoes together with a smoothly decreasing background term. This background arises because the pump pulse raises the temperature of the $\mathrm{Al}$ film, thereby giving a change in optical reflectivity proportional to the temperature rise $\Delta \Theta$ (thermoreflectance). The temperature change relaxes as heat is conducted into the substrate and this causes the steady decrease in $\Delta R(t)$. The cooling of the Al film is determined by the Kapitza conductance $\sigma_{K}\left(=1 / R_{K}\right)$ together with the thermal conductivity of the material the $\mathrm{Al}$ is deposited on. By analysis of the cooling curve it is possible to determine the value of $\sigma_{K}$, and we have developed a computer program to carry out the necessary fitting procedures.

At the time of submission of our previous proposal we had just made some measurements of this type for gold films deposited onto three alkali halide crystals $\left(\mathrm{LiF}, \mathrm{CaF}_{2}\right.$ and $\mathrm{BaF}_{2}$ ). The surfaces of these crystals had been cleaved prior to deposition of the Au films, and the films were evaporated at pressures in the range $\sim 10^{-6}$ torr. Values obtained for $\sigma_{K}$ were in the range around $10^{4} \mathrm{~W} \mathrm{~cm}^{-2} \mathrm{~K}^{-1}$, and thus the thermal impedance of 
the interface is equivalent to the impedance of an aluminium layer of thickness about $2 \mu$. The values found for $\sigma_{K}$ were in reasonable agreement with calculations based upon a simple lattice dynamical model [3]. In this model the $\mathrm{Al}$ and the alkali halide were approximated by fcc lattices in which the atoms interacted by nearest-neighbor central forces. The strength of these forces was chosen so as to give the correct Debye temperature for both materials.

We had planned to extend this work in two ways. We proposed to study $\sigma_{K}$ at interfaces that were prepared under better-defined conditions using UHV techniques. Secondly, we planned to investigate some anomalous effects seen in the cooling curves when the temperature was in the range around $100 \mathrm{~K}$, that we believe are due to second sound in the substrate. However, before embarking on these projects we became aware of the remarkable results obtained at General Electric Company on isotopically-pure synthetic diamond crystals. Room temperature measurement of the thermal conductivity of these crystals gave the result $33 \mathrm{~W} \mathrm{~cm}^{-1} \mathrm{~K}^{-1}$, i.e. $50 \%$ larger than the accepted value for natural diamond and more than 8 times larger than copper. The explanation of this very large conductance is of fundamental interest, and also synthetic diamond may be an attractive material for use as a heat-sink in high power semiconductor circuits.

We realised that it was important to measure the Kapitza resistance between diamond and other materials. In the first place if this Kapitza resistance was very large, the practical gains to be obtained from the high bulk thermal conductivity would be limited. Secondly, one might well expect that heat flow between ordinary materials and diamond might be interesting because of the unusual lattice dynamics of diamond. The Debye temperature of diamond is $2200 \mathrm{~K}$, and hence the major part of its phonon spectrum lies above the upper limit of the phonon spectrum in conventional materials. Thus it appeared that a large fraction of the phonons in diamond had too high a frequency to be be transmitted across an interface to another material, and so $R_{K}$ should be high.

Dr William Banholzer of GE provided us with several diamond samples and we set out to measure $\sigma_{K}$ between diamond and several other materials ( $\mathrm{Ti}, \mathrm{Al}, \mathrm{Au}$ and $\mathrm{Pb}$ ). The results are described in the attached reprint [13]. The conductivity was found to decrease in the sequence $\mathrm{Ti}, \mathrm{Al}, \mathrm{Au}$, and $\mathrm{Pb}$, and this is the trend that one could expect based on the Debye temperatures $\Theta_{D}$, i.e. based on the idea that $\sigma_{K}$ would be smallest for the combination of materials with the biggest difference in Debye temperatures. However, the variation from metal to metal was much smaller than expected based on the lattice dynamical theory described above, and for $\mathrm{Pb}$ the experimentally-measured $\sigma_{K}$ is about 100 times the theoretical value at room temperature! In addition, the measured $\sigma_{K}$ violates an upper bound on the conductivity usually called the radiation limit. This limit is obtained by supposing that all phonons coming from the diamond side of the interface that have frequencies less than the upper frequency limit $\nu^{\max }$ in the metal are transmitted into the metal with probability 1 . Phonons above this frequency are supposed to be reflected. This limiting value of $\sigma_{K}$ is substantially below the experimental values for $\mathrm{Pb}$, and this implies that there must be a large contribution to the conductance from phonons with frequency $\nu$ greater than $\nu_{P b}^{\max }$, the maximum frequency in the $\mathrm{Pb}$. 
We have considered several possible explanations of this. One possible mechanism could arise from the transfer of energy from phonons in the diamond with $\nu>\nu_{P b}^{\max }$ transferring energy directly to the conduction electrons in the $\mathrm{Pb}$. This process has been considered in the past in theories of the Kapitza conductance between a metal and liquid helium. Within the last two months, we have tested this idea by measuring $\sigma_{K}$ to $\mathrm{Bi}$. Bismuth has a very low density of free electrons but has an atomic mass and a $\Theta_{D}$ similar to those of $\mathrm{Pb}$. We found that $\sigma_{K}$ was similar for $\mathrm{Bi}$ and $\mathrm{Pb}$, thus implying that a coupling to the electrons is not the dominant process. Another possibility is anharmonicity in the $\mathrm{Pb}$. This means that a single phonon coming from the diamond might excite two or more phonons in the $\mathrm{Pb}$, and in the reverse process two or more phonons in the $\mathrm{Pb}$ could combine to create a high energy phonon in the diamond. To investigate these processes quantitatively we have recently performed molecular dynamics simulations of the heat flow across the interface. In these calculations we consider the flow of heat from the $\mathrm{Pb}$ into the diamond, and use a Lennard-Jones potential to model the interatomic forces in the $\mathrm{Pb}$. To save on computer time the calculations are performed in two dimensions. The results at the present time are very encouraging and indicate that anharmonicity can indeed give an enhancement large enough to explain the experimental results.

In addition to the Kapitza conductance to diamond, we have studied the conductance to $\mathrm{BaF}_{2}$ and sapphire. For $\mathrm{BaF}_{2}$ the results are in reasonable agreement with the lattice dynamical theory. For $\mathrm{Pb}$ and $\mathrm{Au}$ on sapphire, however, the conductance is much larger than expected on the lattice dynamical model. This is perhaps not surprising considering that sapphire, like diamond, has a high Debye temperature $(\sim 1000 \mathrm{~K})$.

\section{e) THEORETICAL WORK}

In addition to these projects, we have performed several calculations on the propagation of phonons in crystals. In one calculation [5] we considered what happens when high energy phonons are introduced into a crystal at $T=0 \mathrm{~K}$, and propagate while subject to isotope scattering and anharmonic decay processes. In the limit of very strong isotope scattering it has been shown by Levinson and co-workers that the phonons undergo "quasidiffusion". This means that their frequency spectrum and their spatial distribution obey scaling laws, and that the rate at which they diffuse increases as a power of the time. We were able to establish the range of validity of these scaling laws and to discuss the extent to which they should apply to real crystals. Very recently, J.P. Wolfe and his students at the University of Illinois have performed some experiments to test our theoretical ideas, and have obtained good agreement with our results. In another calculation[12], with S. Tamura (Hokkaido University, Japan), we have investigated the anharmonic decay rate for phonons in a crystal at $T=0 \mathrm{~K}$, and found the range of wave vectors for which phonons are stable against anharmonic decay. As well as being of fundamental interest, this range is relevant to current attempts to detect dark matter particles using phonon-based crystal detectors. In a paper to be submitted to the phonon conference this summer we have calculated the velocity of second sound in crystals as a function of temperature [23]. We find that as the 
temperature is raised above $0.1 \Theta_{D}$ the second sound velocity drops substantially below its low temperature limiting value. This decrease in velocity is of considerable significance for the analysis of the thermal conductivity of crystals, and may resolve some long--standing puzzles about the relative strength of Umklapp and normal phonon scattering processes. Finally, with K. Yakubo and T. Nakayama we performed an analysis of a method we had earlier proposed for the determination of the phonon eigenmodes of disordered crystals (see attached reprint [11]).

\section{PUBLICATIONS RESULTING FROM RESEARCH SUPPORTED BY THIS GRANT}

At the time of submission of our previous proposal, abstracts of the following four papers had been submitted to the Phonons '89 conference in Heidelberg, August, 1989.

[1] Picosecond Optics Measurement Attenuation of High Frequency Phonons in a-SiO ${ }_{2}$, C.D. Zhu, H.J. Maris, and J. Tauc, in Proceedings of Phonons '89, edited by S. Hunklinger, W. Ludwig, and G. Weiss (World, Singapore, 1988), p. 498.

[2] Picosecond Optics Studies of the Kapitza Resistance Between Solids at High Temperature, R. Stoner, D.A. Young, H.J. Maris, and J. Tauc, in Proceedings of Phonons '89, edited by S. Hunklinger, W. Ludwig, and G. Weiss (World, Singapore, 1988), p. 1305.

[3] Theory of Kapitza Conductance Between Solids at High Temperatures, D.A. Young and H.J. Maris, in Proceedings of Phonons '89, edited by S. Hunklinger, W. Ludwig, and G. Weiss (World, Singapore, 1988), p. 1329.

[4] Phonon Attenuation and Velocity Measurements in Fused Quartz by Picosecond Acoustic Interferometry, H.-N. Lin, H.J. Maris, J. Tauc, and H.T. Grahn, in Proceedings of Phonons '89, edited by S. Hunklinger, W. Ludwig, and G. Weiss (World, Singapore, 1988), p. 1430.

[5] Phonon Proragation with Isotope Scattering and Anharmonic Decay, H.J. Maris, Phys. Rev. 41, 9736 (1990).

[6] Non-Destructive Testing of Microstructures by Picosecond Ultrasonics, H.-N. Lin, H.J. Maris, and R.J. Stoner, J. Non-Destructive Evaluation 9, 239 (1990).

[7] Quantum Acoustics, H.J. Maris, invited article in McGraw-Hill Encyclopaedia of Science and Technology (to be published).

[8] Phonon Attenuation and Velocity Measurements in Transparent Materials by $P_{\hat{\imath}}$ cosecond Acoustic Interferometry, H.-N. Lin, R.J. Stoner, H.J. Maris, and J. Tauc, J. Appl. Phys. 69, 3816 (1991).

[9] Attenuation of Longitudinal Acoustic Phonons in Amorphous $\mathrm{SiO}_{2}$ at Frequencies up io 440 GHz, C.-D. Zhu, H.J. Maris, and J. Tauc, Phys. Rev. Lett. 66, 45 (1991).

[10] Ultrasonic Experiments at Ultra-High Frequency with Picosecond Time-Resolution, H.-N. Lin, R.J. Stoner, and H.J. Maris, in Proceedings of the IEEE 1990 Ultrasonics Ultrasonics Symposium, editor B.R. McAvoy (IEEE, New Jersey, 1991), p. 1301. 
[11] Analysis of a New Method for Finding Eigenmodes of Very Large Lattice Systems, K. Yakubo, T. Nakayama, and H.J. Maris, J. Jap. Phys. Soc. 60, 3249 (1991).

[12] Anharmonic Decay and the Propagation of Phonons in an Isotopically Pure Crystal at Low Temperatures, H.J. Maris and S. Tamura, submitted to Physical Review.

[13] Measurements of the Kapitza Conductance Between Diamond and Several Metals, R.J. Stoner, H.J. Maris, T.R. Anthony, and W.F. Banholzer, Phys. Rev. Lett. 69, 1563 (1992).

[14] Detection of Titanium Silicide Formation and Phase Transformation by Picosecond Ultrasonics, H.-N. Lin, R.J. Stoner, H.J. Maris, J.M.E. Harper, C. Cabral, J.-M. Halbout, and G.W. Rubloff, to be published in the Proceedings of the San Francisco Meeting of the Materials Research Society, April 1992.

[15] Setection of Thin Interfacial Layers by Picosecond Ultrasonics, G. Tas, R..J. Stoner, H.J. Maris, G.W. Rubloff, G.S. Oehrlein, and J.M. Halbout, to be published in the Proceedings of the San Francisco Meeting of the Materials Research Society, April 1992.

[16] Non-Destructive Detection of Titanium Di-Silicide Phase Transformation by Picosecond Ultrasonics, H.-N. Lin, R.J. Stoner, H.J. Maris, J.M.E. Harper, C. Cabral, J.-M. Halbout, and G.W. Rubloff, submitted to Applied Physics Letters.

[17] Picosecond Ultrasonic Investigation of Thin Interfacial Layers Between Metal Films and Silicon, G.Tas, R.J. Stoner, H.J. Maris, G.W. Rubloff, G.S. Oehrlein, and J.M. Halbout, submitted to Applied Physics Letters.

[18] Study of Vibrations of Gold Nanostructures by Picosecond Ultrasonics, H.-N. Lin, H.J. Maris, J. Tauc, K.Y. Lee, H. Luhn, and D.P. Kern, submitted to Journal of Applied Physics.

[19] Picosecond Optics Studies of Vibrational and Mechanical Properties of Nanostructures, H.J. Maris, H.-N. Lin, C.J. Morath, R.J. Stoner, and G.I Tas, invited paper to appear in the Proceedings of the Acousto-optics and Acoustic Microscopy Symposium at the American Society of Mechanical Engineers winter meeting, Anaheim, California, November, 1992.

[20] Anharmonic Decay and the Propagation of Phonons in an Isotopically Pure Crystal at Low Temperatures: Application to Dark Matter Detection., H.J. Maris and S. Tamura, submitted to Physical Review.

Abstracts for the following papers have been submitted to the 7 th International Conference on Phonon Scattering in Condensed Matter, to be held at Ithaca, New York, August, 1992. The papers are at various stages of preparation. Paper [25] is an invited review.

[21] Picosecond Optical Study of the Kapitza Conductance Between Metals and Dielectrics at High Temperature, R.J. Stoner and H.J. Maris.

[22] Molecular Dynamical Simulations of the Kapitza Conductance Between Harmonic and Anharmonic Lattices, R.J. Stoner and H.J. Maris. 
[23] Theory of Second Sound in Crystals at High Temperature, R.J. Stoner and H.J. Maris.

[24] Measurement of Acoustic Attenuation at High Frequencies Using Surface Modes in Multilayers, C.J. Morath, R.J. Stoner, and H.J. Maris.

[25] Picosecond Studies of Phonons, H.J. Maris.

\section{Ph.D. Theses}

C.-D. Zhu, Studies of Phonons in Disordered Systems Using Picosecond Optical Techniques, September 1991.

H.-N. Lin, Study of Thin Films and Nanostructures by Picosecond Ultrasonics, May 1992.

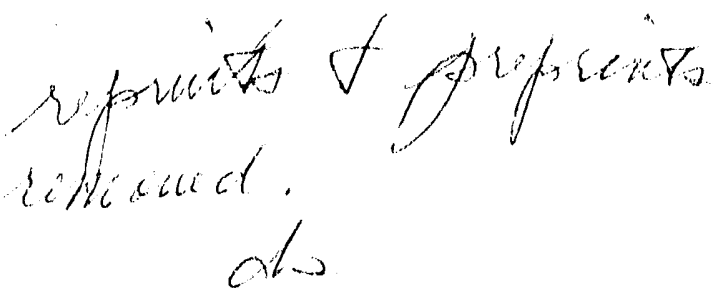




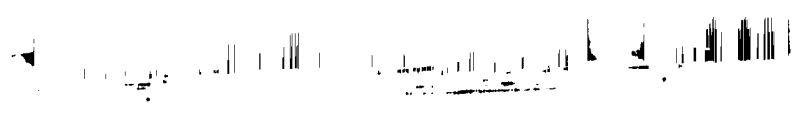

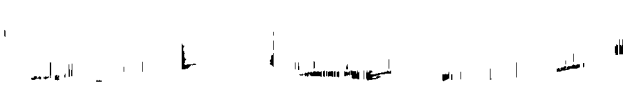

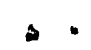
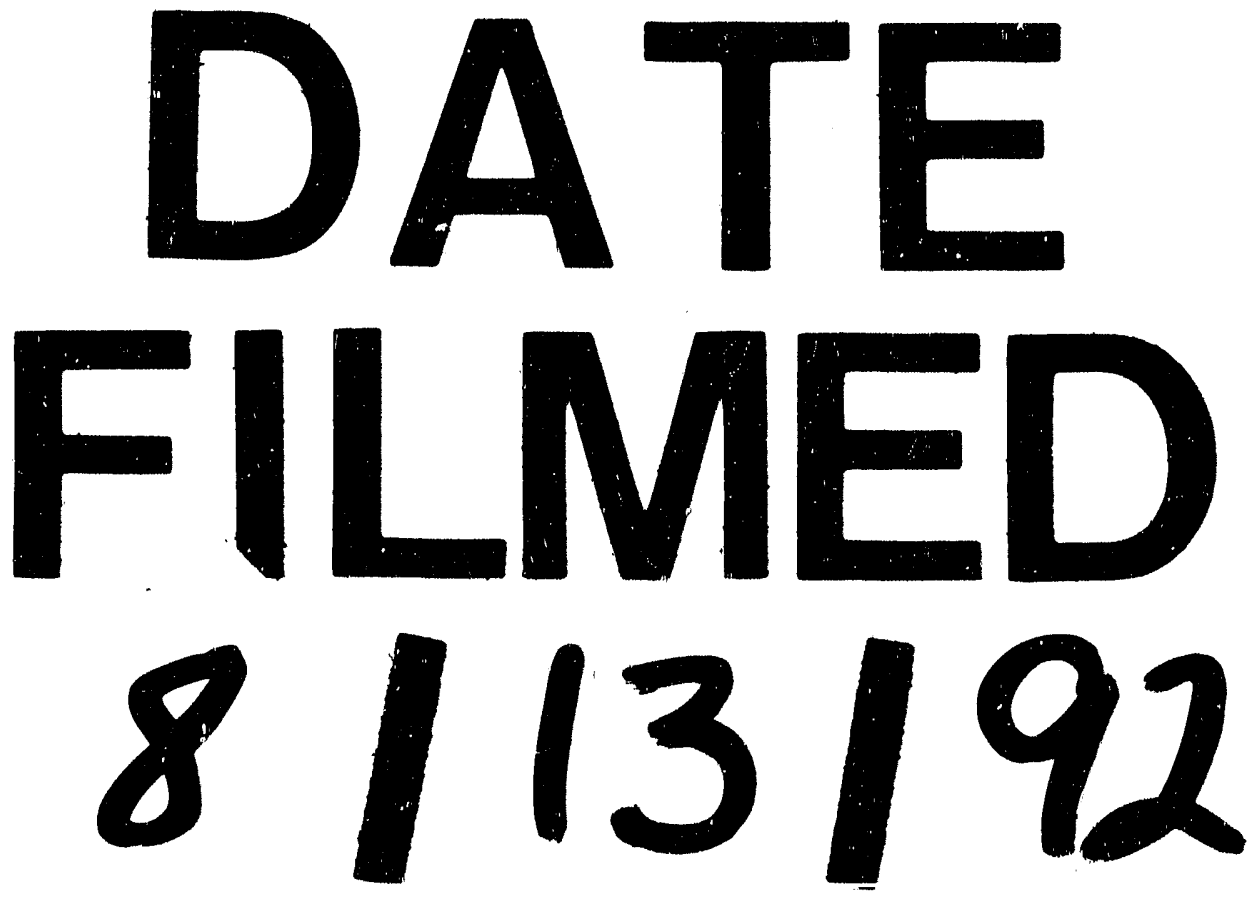
